Trauma Berufskrankh 2014 • 16[Suppl 2]:146-151 DOI 10.1007/s10039-013-2016-8

Online publiziert: 14. November 2013

(c) Springer-Verlag Berlin Heidelberg 2013

\title{
U. Brunner
}

Abteilung für Unfall-, Schulterchirurgie, Krankenhaus Agatharied, Hausham

\section{Posttraumatische Rotatoren- manschettenläsionen}

die Sehnenstruktur von innen verändern. Dies sind u. a. verminderte Durchblutung, abnehmende Elastizität und Alterungsvorgänge. Nachgewiesen sind auch der negative Effekt von Adipositas und Nikotin sowie genetische Faktoren [25].

Extrinsisch sind v. a. Faktoren, die nach Neer [17] dem „outlet impingement“ zugeordnet werden können. Dies sind u. a. der Akromionsporn und kaudale Osteophyten am Akromioklavikulargelenk. Später wurde auch die laterale Breite des Akromions als Kofaktor für häufigere RM-Läsionen nachgewiesen [18].

Auch am M. subscapularis wurden degenerative Partialläsionen in gleicher Häufigkeit wie am M. supraspinatus gefunden. Bei Vorliegen einer gelenkseitigen Partialläsion waren die Sehnen eher degenerativ verändert [24]. Für die Subskapularissehnen wurde als extrinsischer Mechanismus eine gelenkseitige Faserüberdehnung bei Druck durch den Processus coraoideus von außen postuliert [TUFFLäsion (TUFF: ,tensile under-surface fiber failure“), [8]].

Extrinsische Ursachen können auch repetitive Mikrotraumen sein, die heute als Auslöser für degenerativ entzündliche Prozesse angesehen werden [27]. Makrotraumatische Faktoren sind von außen einwirkende Kräfte, die entweder durch direktes Trauma, als exzentrische Belastung angespannter Sehnen oder als Scherkräfte bei Luxationen vorkommen können.

Die intrinsischen Faktoren gelten heute als die überwiegende Ursache für Veränderungen oder Rupturen der Rotatorenmanschette.

\section{Verletzungsmechanismen}

\section{Festigkeit und Steifheit der Sehne,} einwirkende Kraftvektoren

Vor dem Hintergrund einer hohen Inzidenz alters- und verschleißassoziierter Veränderungen der Rotatorenmanschette wird die traumatische Genese einer Rotatorenmanschettenläsion häufig negativ diskutiert. Da die degenerativen Veränderungen besonders die Supraspinatussehne betreffen, wird von manchen Gutachtern angenommen, dass eine gesunde $\mathrm{Su}$ praspinatussehne nicht reißen könne und damit die traumatische Genese einer isolierten Supraspinatussehnenruptur in der Regel ausgeschlossen sei [11].

Die Reißfestigkeit der Supraspinatussehne wurde nur in wenigen Studien überprüft. Biomechanische Untersuchungen $24 \mathrm{~h}$ postmortal zeigten eine signifikante Korrelation der Zugfestigkeit und der Steifigkeit der Supraspinatussehne mit dem Alter. Während die Sehne eines Jugendlichen einen Maximalwert von $1850 \mathrm{~N}$ aufwies, zeigte eine 65 Jahre alte Sehne eine maximale Zugfestigkeit von über $900 \mathrm{~N}$ und muss nicht zwangsläufig eine Ruptur aufweisen [22].

Bei der Analyse von außen einwirkender Kräfte müssen direkte und exzentrisch einwirkende Kräfte unterschieden werden. Je weiter peripher eine Kraft am Arm ansetzt, umso größer ist die zentral einwirkende Kraft. So kann eine Last, die mit $5 \mathrm{~kg}$ an der ausgestreckten Hand einwirkt, ohne reflektorische Gegenspannung eine Hebelkraft von $1500 \mathrm{~N}$ auf den Ansatz der RM entfalten [9]. Darüber hinaus können in Abhängigkeit von der Arm- 


\begin{tabular}{|c|c|c|}
\hline & Pro & Kontra \\
\hline $\begin{array}{l}\text { Alter, } \\
\text { Vorerkrankungen }\end{array}$ & $\begin{array}{l}<40 \text { Jahre } \\
\text { Keine Vorerkrankung }\end{array}$ & Vorgeschichte RM-Schaden \\
\hline Unfall & $\begin{array}{l}\text { Unfallereignis/Unfallmecha- } \\
\text { nismus }\end{array}$ & Kein Unfall (Gegenbeweis) \\
\hline Primärbefund & $\begin{array}{l}\text { Hämatom, ,,drop arm", } \\
\text { Außenrotationsschwäche }\end{array}$ & Negativ \\
\hline Arztbesuch & $<3$ Tage & Kein Arztbesuch $<2$ Wochen \\
\hline \multirow[t]{5}{*}{ Befunde } & & $\begin{array}{l}\text { Muskelatrophie } \\
\text { Alte Bizepssehnenruptur }\end{array}$ \\
\hline & $\begin{array}{l}\text { Röntgen: keine Sekundär- } \\
\text { veränderungen }\end{array}$ & $\begin{array}{l}\text { Röntgen: Sekundärveränderungen (HK, AK) } \\
\text { Humeruskopfhochstand }\end{array}$ \\
\hline & $\begin{array}{l}\text { Sonographie }<2 \text { Wochen: } \\
\text { Hämatom }\end{array}$ & \\
\hline & MRT <6 Wochen: Ödem & MRT $<12$ Wochen: fettige Degeneration \\
\hline & & Gegenseite? Röntgen, Sonographie, Klinik \\
\hline Operation & $\begin{array}{l}\text { Hämarthros, Rupturform } \\
\text { nicht abgerundet }\end{array}$ & $\begin{array}{l}\text { Situs } \\
\text { Kein Hämarthros }\end{array}$ \\
\hline Histologie & Typische Befunde & Typische Befunde \\
\hline
\end{tabular}

stellung sehr unterschiedliche Kraftvektoren auf die einzelnen anatomischen Strukturen einwirken [20]. Diese Effekte sind in der Einzelanalyse der Verletzung nicht nachvollziehbar. Die Kraft wird außerdem rasch ansteigen, wenn die Grundlänge des Muskels überschritten wird. Die Muskeln der Rotatorenmanschette haben einen großen Querschnitt, aber nur eine relativ kleine Verkürzungsstrecke, d. h. sie sind sehr stark. Fibrotische Umbauvorgänge verringern ihre Elastizität zusätzlich [9].

Nach experimenteller Trennung in eine gelenk- bzw. bursaseitige Schicht verringerte sich die Zugfestigkeit auf $500 \mathrm{~N}$ [15]. Nach einer anderen Untersuchung waren die vorderen Anteile der Supraspinatussehne stärker als die hinteren, insgesamt aber nicht sehr stark (652 N; [7]).

\section{Für eine Sehnenzerreißung ungeeignete und geeignete Unfallmechanismen}

Da die physiologische Relativbewegung des Oberarmkopfs zur Pfanne gering ist, können bei einem direkten Anpralltrauma oder beim Sturz nach vorn bzw. seitlich auf den ausgestreckten Arm oder Ellenbogen ohne starke Verdrehung oder forcierte Adduktion keine wesentlichen Kräfte auf die Sehnen der Rotatorenmanschette einwirken. Auch die aktive Kraftanstrengung beim kontrollierten Anhe- ben und kontinuierlicher Krafteinleitung ist hierfür ungeeignet.

Grundsätzlich in der Lage, eine ZerreiBung der Rotatorenmanschette herbeizuführen, sind zum einen Mechanismen, die mit einer Luxation verbunden sind und damit im Sinne der Abscherung von innen wirken, zum anderen Mechanismen, die eine starke exzentrische Krafteinleitung auf die angespannte Rotatorenmanschettenmuskulatur bewirken.

Die vordere Schulterluxation ist mit einer Prävalenz von 33,4\% mit einer Rotatorenmanschettenläsion verbunden [23]. Die hintere Schulterluxation, die allerdings sehr selten ist [12], kann ebenfalls zu einer Rotatorenmanschettenzerreißung führen. Der Verletzungsmechanismus ist eine direkte Krafteinleitung auf den nach vorne ausgestreckten, adduzierten und innenrotierten Arm. Geeignet sind auch eine passiv forcierte Außenoder Innenrotation auf das angespannte Schultergelenk, z. B. beim Versuch, sich an einer Tür oder einer Leiter während des Fallens zu halten, ebenso die forcierte passive Traktion nach unten, vorne oder hinten, z. B. beim Versuch, einen fallenden schweren Gegenstand aufzuhalten, bei dem der Oberarmkopf aus der Pfanne herausgezogen wird. Der Sturz auf den ausgestreckten und außenrotierten Arm beim Abstützen nach hinten führt zu einer axialen Krafteinleitung nach vorne und oben und wird ebenfalls als geeigneter Mechanismus angesehen [13].

\section{Symptomatik und natürlicher Verlauf}

Chronische Rotatorenmanschettenläsionen können, v. a. wenn sie biomechanisch kompensiert sind, relativ asymptomatisch verlaufen. Durch entzündlich degenerative Prozesse, die gelegentlich auch durch ein direktes oder indirektes Trauma entstehen, können sich aber auch allmählich stark schmerzhafte Bewegungseinschränkungen entwickeln.

Die traumatische Rotatorenmanschettenruptur hat dagegen einen typischen Verlauf. Das plötzlich einwirkende Unfallereignis führt unmittelbar zu einem deutlichen, oft reißenden Schmerz in der Schulter mit Ausstrahlung in den Oberarm, verbunden mit einem Kraft- und Funktionsverlust (Pseudoparalyse). Äußere Verletzungszeichen, wie Hämatom oder Schwellung, können, müssen aber nicht vorhanden sein [5]. Aufgrund der deutlichen Klinik wird sich der Patient in der Regel bald einem Arzt vorstellen. Nach Rückgang des akuten Schmerzes sind aktive, später aber auch passive Bewegungseinschränkungen nachweisbar. Die spezifischen Rotatorenmanschettenzeichen sind bei der klinischen Untersuchung schmerzhaft und positiv. Nach Rückgang der subakuten Phase (3 bis 14 Tage) wird sich zunehmend eine passive Steifigkeit einstellen (postakute Phase). Der klinischen, aber auch der radiologischen Diagnostik kommt damit eine wichtige differenzialdiagnostische Rolle zu [9].

Trotz der Hinweise, dass auch degenerative Veränderungen in gleicher Anzahl an der Subskapularissehne nachzuweisen sind, gelten diese weiterhin als mehrheitlich traumatisch bedingt [9].

\section{Klinische und radiologische Diagnostik}

Zur primären Diagnostik ist die Durchführung einer Röntgenaufnahme in Truea.p.-, Outlet- und axialer Projektion erforderlich. Nach einer frischen Verletzung besteht in der Regel kein Humeruskopfhochstand. Eine Verminderung 
des akromiohumeralen Abstandes entwickelt sich auch bei einer großen Rotatorenmanschettenruptur erst allmählich [4]. Sekundäre degenerative Veränderungen können auf eine vorbestehende Rotatorenmanschettenruptur hinweisen. Diese sind ein Os acromiale, ein Akromionsporn in der Outlet-Aufnahme, eine Hypersklerosierung oder Abrundung des Tuberculum majus oder ein deutlich nach lateral überhängendes Akromion (lateraler Akromionindex, [18]). Der Nachweis begleitender Frakturen, z. B. an der Pfanne (Bankart-Fraktur), am Tuberculum majus oder minus oder eine Hill-SachsImpressionsfraktur am Kopf sind beweisend für traumatische Ereignisse [26, 28].

Neben der Sonographie ist heute v. a. die Magnetresonanztomographie (MRT) ein probates Mittel, um die traumatische Genese der Rotatorenmanschettenläsion nachzuweisen. Gerade im Röntgenbild nicht nachweisbare Frakturen am Tuberculum majus oder an der Kalotte können als „bone bruise“ innerhalb der ersten 6 Wochen richtungweisend sein. Eher typisch für traumatische Läsionen sind kombinierte Verletzungen der Subskapularis- und Supraspinatus- bzw. der Supraspinatus- und Infraspinatussehne [13]. Typisch und als indirekter Hinweis auf eine traumatische Läsion gelten auch begleitende Nervenläsionen, die klinisch primär erkannt und registriert werden müssen [21].

Im MRT sind aber auch Argumente gegen die traumatische Genese der Rotatorenmanschettenläsion abzuklären. Diese sind u. a. das Fehlen des subakromialen Fettzeichens, degenerative und dünne Sehnen der Rotatorenmanschette, eine fettige Degeneration der begleitenden Muskulatur bzw. Atrophie sowie der Nachweis einer bilateralen Rotatorenmanschettenruptur. Der Nachweis einer Tendinosis calcarea ist dagegen nicht sicher als Hinweis auf eine vorbestehende Rotatorenmanschettenläsion zu werten [10].

\section{Beurteilung der Genese - Pro und Kontra}

Da die intrinsischen Vorschäden bei zunehmendem Alter häufig und die Verletzungsmechanismen schwierig abzugren-

Trauma Berufskrankh 2014 - 16[Suppl 2]:146-151 DOI 10.1007/s10039-013-2016-8

(c) Springer-Verlag Berlin Heidelberg 2013

U. Brunner

\section{Posttraumatische Rotatorenmanschettenläsionen}

\section{Zusammenfassung}

Hintergrund. Die zunehmende Häufigkeit von Rotatorenmanschettenläsionen im Alter ist ein Hinweis, dass diese meist Folge eines intrinsischen, natürlichen Alterungsvorgangs sind. Abzugrenzen sind extrinsische Schäden, die durch repetitive Mikro- oder Makrotraumen ausgelöst werden. Während direkte Traumen in der Regel die starken Sehnen nicht schädigen können, führen exzentrische Belastungen angespannter Sehnen oder Scherkräfte bei Luxationen zu traumatischen Rotatorenmanschettenrupturen.

Beurteilung. Die Entscheidung, ob es sich um eine traumatische Sehnenruptur handelt, fällt in einer individuellen patientenbezogenen Analyse aller begleitenden Faktoren.
Hierbei sind der Zeitverlauf, der primäre Befund sowie Röntgen- und Magnetresonanztomographieaufnahmen einzubeziehen. Therapie. Die Indikation zur Operation besteht v. a. bei jüngeren Patienten mit akuten, unfallbedingten bzw. im Rahmen einer Luxation entstandenen Rupturen. Die Versorgung erfolgt frühzeitig. Offene und arthroskopische Techniken ergeben gute bis sehr gute Ergebnisse, auch im Langzeitverlauf.

\section{Schlüsselwörter}

Rotatorenmanschette .

Schulterimpingementsyndrom - Trauma ·

Operative Behandlung · Arthroskopie

\section{Posttraumatic rotator cuff tears}

\section{Abstract}

Background. The increasing incidence of rotator cuff tears with increasing age indicates that rotator cuff lesions are naturally correlated with ageing and often present without clinical symptoms. Rotator cuff tears from extrinsic causes, i.e. repetitive microtrauma or even macrotrauma, are different entities. Because of the strong and stiff tendon tissue, direct injury mechanisms may not lead to a rotator cuff tear. Excentric mechanisms of tensioned tendons or shearing forces during dislocation may, on the other hand, result in traumatic rotator cuff tears.

Evaluation. For the decision from a legal point of view of whether it is a traumatic or degenerative rotator cuff tear, an indi- vidual- and patient-related analysis of all circumstances and information including radiographs or MRI is necessary.

Therapy. Reconstruction of the tear is indicated especially in younger patients as well with a history of relevant trauma or of dislocation. The operation should be performed early, if associated with concomitant neurologic lesions. Good to very good results are achieved using open or arthroscopic techniques in the short and long-term follow-up.

\section{Keywords}

Rotator cuff · Shoulder impingement syndrome · Trauma - Operative therapy . Arthroscopy zen sind, ist die gutachterliche Bewertung auf eine Einzelfallbeurteilung angewiesen. Es ergeben sich daher Pro- und Kontraargumente, die mit Wahrscheinlichkeit für oder gegen einen ursächlichen Zusammenhang zwischen einer traumatischen Genese und der nachgewiesenen Rotatorenmanschettenläsion sprechen (• Tab. 1).

\section{Operative Behandlung}

\section{Indikation}

Traumatische Rotatorenmanschettenrupturen sollten bei der Therapieentschei- dung hinsichtlich konservativer oder operativer Versorgung anders bewertet werden als degenerative Rupturen. Die Indikation zur Operation besteht daher v. a. bei jüngeren Patienten mit

- akuten, unfallbedingten Rupturen,

- im Rahmen einer Luxation entstandenen Rupturen,

- Rupturen des M. subscapularis mit oder ohne Luxation der langen $\mathrm{Bi}$ zepssehne und

- bei Kombinationsrupturen der Subskapularis- und Supraspinatussehne (anterosuperiore Ruptur; [16]) bzw. 

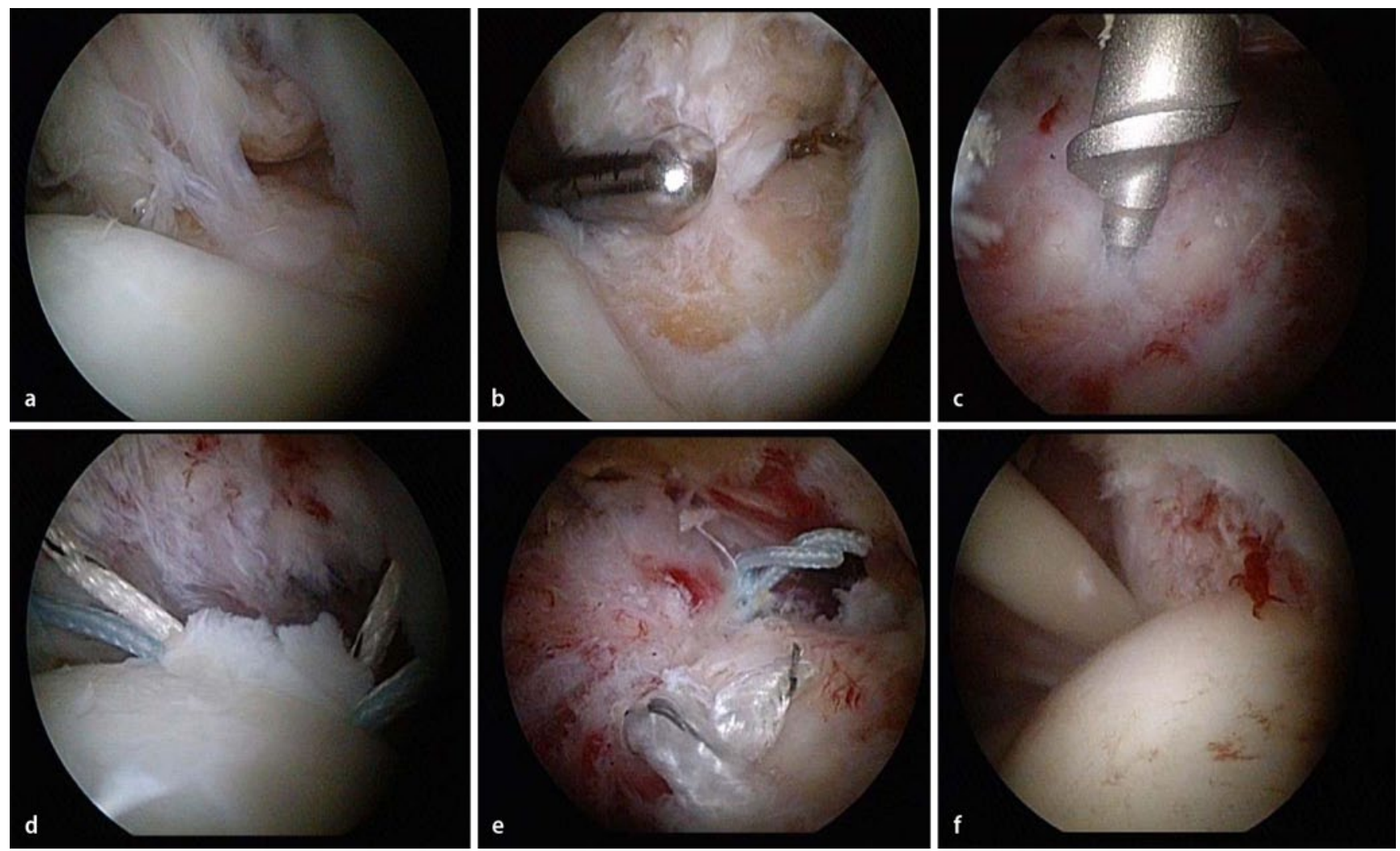

Abb. 1 \ 45-jähriger Patient, traumatische Supraspinatussehnenpartialläsion größer A3 (50\% des Ansatzes), a Läsion, b Débridement, c transtendinöser Anker, d Ausleiten der Fäden, e subakromialer Knoten, f Kontrolle intraartikulär

- bei kombinierten Rupturen der Supra- und Infraspinatussehne (posterosuperiore Rupturen; [25]).

\section{Zeitpunkt der Operation}

In einem systematischen Review der Literatur bei Evidenzlevel 1-4 konnte keine ausreichende Datenlage zur Empfehlung der frühzeitigen Rekonstruktion gefunden werden [13]. Hantes et al. [6] zeigten in einer Level-3-Studie allerdings bessere Ergebnisse hinsichtlich der Funktion gegenüber einer verzögerten Rekonstruktion (3 Wochen nach dem Unfall). Bjornsson et al. [1] konnten keine Korrelation des strukturellen oder klinischen Ergebnisses nach Rekonstruktion von traumatischen Rotatorenmanschettenrupturen innerhalb der ersten 3 Monate finden. Petersen u. Murphy [19] fanden sogar keinen Unterschied innerhalb der ersten 4 Monate nach dem Unfall.

Je jünger ein Patient und je wahrscheinlicher die traumatische Genese, z. B. nach Luxation, tendieren wir zur möglichst frühzeitigen Rekonstruktion.
Dies gilt insbesondere auch, wenn begleitende neurologische Läsionen eine längere Erholungsphase erwarten lassen.

Während Rupturen der Supraspinatussehne nach verzögerter Rekonstruktion häufig gute Ergebnisse bieten, wurde für die verzögerte Rekonstruktion der Subskapularissehnenruptur ein schlechteres klinisches Ergebnis nachgewiesen [3].

\section{Versorgungstechniken und Ergebnisse der Rekonstruktion posttraumatischer Läsionen}

Gedeckte Läsionen des Rotatorenintervalls („hidden lesions“) bzw. Partialläsionen der Supraspinatussehne sind sehr gute Indikationen für eine arthroskopische transtendinöse Versorgung ( $\mathbf{D}$ Abb. 1). Hierbei müssen die Sehnen nicht komplett von ihrem Ursprung abgelöst werden, sondern können nach Anfrischen des Tuberculum majus transtendinös refixiert werden.

Die Ergebnisse nach arthroskopischer transtendinöser Rekonstruktion sind ausgezeichnet [2]. In einer retrospektiven
Analyse von 30 konsekutiven Patienten mit anterosuperioren, posttraumatischen Rotatorenmanschettenrupturen und offener Rekonstruktion im Mittel 4 1/2 Monaten nach dem Trauma zeigten sich signifikante Verbesserungen der funktionellen objektiven und subjektiven Ergebnisse (Constant-Score: 93,4\%). Bei Patienten mit anterosuperiorer Rotatorenmanschettenläsion fiel präoperativ v. a. die Innenrotationsschwäche mit begleitender $\mathrm{Bi}$ zepssehnenläsion auf. Größere Defekte, die auch den M. infraspinatus betrafen, zeigten schlechtere Ergebnisse. Die Autoren folgerten, dass durch eine rechtzeitige Diagnose und eine frühzeitige offene Rekonstruktion nahezu normale Schulterfunktionen zu erreichen sind [16].

Eigene Beobachtungen bei kombinierten anterosuperioren Läsionen ergaben in der Regel typische erhaltene Sehnenstrukturen als Verbindung zwischen Subskapularis- und Supraspinatussehne (Kommazeichen). Diese Läsionen sind operativ gut zu versorgen, da trotz der weiten Retraktion der Zweisehnenverletzung in der Regel eine gute Rekonstruktion und sichere 


\section{Schulterverletzungen}
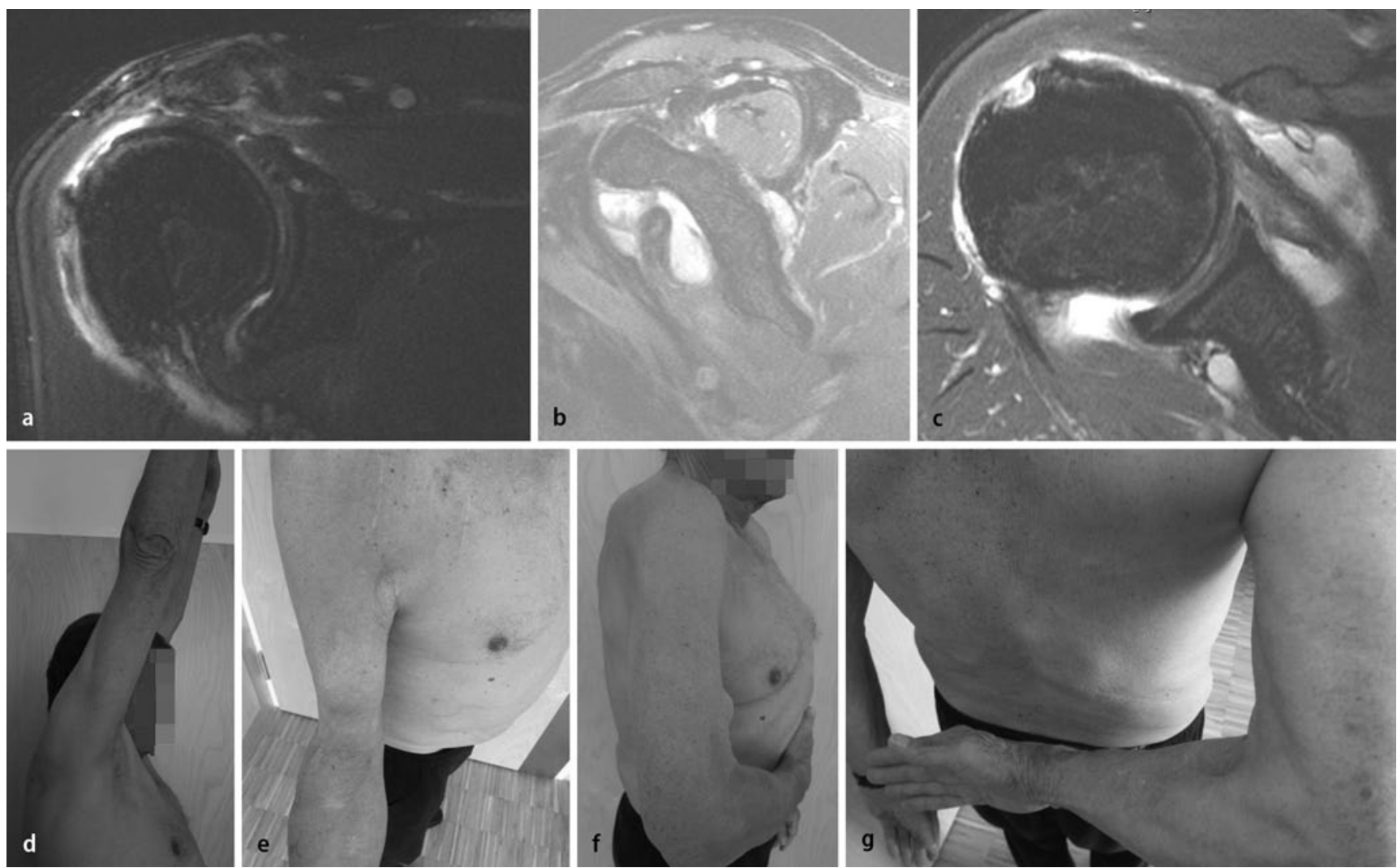

Abb. $2 \Delta$ 59-jähriger Patient, traumatische Zweisehnenruptur (a-c), Versorgung durch offene Rekonstruktion und Funktion nach 11 Jahren (d-g), a-c posttraumatische, präoperative MRT (Magnetresonanztomographie), a parakoronar, b parasagittal, $\mathbf{c}$ axial, $\mathbf{d} 11$ Jahre postoperativ, aktive anteriore Elevation, e aktive Außenrotation, $\mathbf{f}$ Belly-Press-Test negativ, $\mathbf{g}$ Lift-off-Test negativ

Verankerung gelingen ( $\mathbf{0}$ Abb. 2). Unterschiede nach arthroskopischer oder offener Rekonstruktion sind im Langzeitverlauf nicht erwiesen.

In einem systematischen, evidenzbasierten Review der Literatur konnten 9 Studien zu traumatischen Rotatorenmanschettenläsionen identifiziert werden. Das durchschnittliche Patientenalter lag zwischen 34 und 61 Jahren, das Intervall bis zur Operation betrug 66 Tage (3 bis 48 Wochen). Der häufigste Verletzungsmechanismus war der Sturz auf den ausgestreckten Arm. Die Supraspinatussehne war in $84 \%$, die Infraspinatussehne in $39 \%$ und die Subskapularissehne in $78 \%$ der Verletzungen betroffen. Die Rupturgröße war in $22 \%$ kleiner $3 \mathrm{~cm}$, in $36 \%$ $3-5 \mathrm{~cm}$ und in $42 \%$ sogar über $5 \mathrm{~cm}$ groß. Die aktive anteriore Elevation verbesserte sich durch die operative Rekonstruktion von 81 auf $150^{\circ}$ postoperativ, der objektive Constant-Score betrug 77 Punkte. Hierdurch kann die Indikation zur operativen Rekonstruktion bestätigt werden, eine evidenzbasierte Empfehlung zur frühen Versorgung ergab sich jedoch nicht [13].

\section{Fazit für die Praxis}

- Traumatische Rotatorenmanschettenläsionen lassen sich durch eine individuelle patientenbezogene, klinische und radiologische Diagnostik identifizieren. Für die häufig kombinierten Zweisehnenläsionen sind die typischen Innen- oder Außenrotationsdefizite wegweisend.

- Auch wenn keine klare klinische Evidenz für die Vorteile einer frühzeitigen operativen Versorgung besteht, überwiegen die klinischen Vorteile deutlich, insbesondere bei den Rupturen des M. subscapularis.

- Ein evidenzbasierter Nachweis der Vorteile der arthroskopischen gegenüber der offenen Rekonstruktion gelang nicht.
- Jüngere und traumatische Rupturen, insbesondere des M. subscapularis, sollten innerhalb der ersten Wochen operativ rekonstruiert werden. Hierbei sind gute bis sehr gute klinische Ergebnisse zu erzielen.

- Die gedeckten Läsionen der oberen Subskapularis- bzw. die Partialläsionen der Supraspinatussehne sind sehr gute Indikationen für die transtendinöse arthroskopische Rekonstruktion.

\section{Korrespondenzadresse}

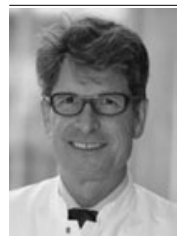

Prof. Dr. U. Brunner

Abteilung für Unfall-, Schulterchirurgie, Krankenhaus Agatharied, Norbert-Kerkel-Platz, 83734 Hausham brunner@khagatharied.de 


\section{Einhaltung ethischer Richtlinien}

Interessenkonflikt. U. Brunner gibt an, dass kein Interessenskonflikt besteht.

Alle Patienten, die über Bildmaterial oder anderweitige Angaben innerhalb des Manuskripts zu identifizieren sind, haben hierzu ihre schriftliche Einwilligung gegeben. Im Falle von nicht mündigen Patienten liegt die Einwilligung eines Erziehungsberechtigen oder des gesetzlich bestellten Betreuers vor.

Dieser Beitrag beinhaltet keine Studien an Menschen oder Tieren.

The supplement containing this article is not sponsored by industry.

\section{Literatur}

1. Bjornsson HC, Norlin R, Johansson K, Adolfsson LE (2011) The influence of age, delay of repair, and tendon involvement in acute rotator cuff tears: structural and clinical outcomes after repair of 42 shoulders. Acta Orthop 82(2):187-192

2. Castagna A, Delle Rose G, Conti M et al (2009) Predictive factors of subtle residual shoulder symptoms after transtendinous arthroscopic cuff repair: a clinical study. Am J Sports Med 37(1):103108

3. Flury MP, John M, Goldhahn J et al (2006) Rupture of the subscapularis tendon (isolated or in combination with supraspinatus tear): when is a repair indicated? J Shoulder Elbow Surg 15(6):659-664

4. Gerber C (1995) Treatment of massive rotator cuff tears. J Bone Joint Surg Br [Suppl II] 77:138

5. Habermeyer P, Lehmann L, Lichtenberg S (2000) Rotatorenmanschetten-Ruptur - Diagnostik und Therapie. Orthopade 29:196-208

6. Hantes ME, Karidakis GK, Vlychou M et al (2011) A comparison of early versus delayed repair of traumatic rotator cuff tears. Knee Surg Sports Traumatol Arthrosc 19(10):1766-1770

7. Itoi E, Berglund LJ, Grabowski JJ et al (1995) Tensile properties of the supraspinatus tendon. J Orthop Res 13:578-584

8. Lo IK, Burkhart SS (2003) The etiology and assessment of subscapularis tendon tears: a case for subcoracoid impingement, the roller-wringer effect, and TUFF lesions of the subscapularis. Arthroscopy 19(10):1142-1150

9. Loew M (2000) Zur traumatischen Entstehung der Rotatorenmanschettenläsion. Wissenschaftliche Grundlagen und ihre Konsequenzen für die Begutachtung. Orthopade 29(10):881-887

10. Loew M, Sabo D, Wehrle M, Mau H (1996) Relationship between calcifying tendinitis and subacromial impingement pathology, a prospective X-ray and MRI study. J Shoulder Elbow Surg 5:314-319

11. Ludolph E, Schröter F, Besig K (1997) Die Begutachtung der Rotatorenmanschettenveränderung. Aktuelle Traumatol 27:31-34

12. Luenam S, Kosiyatrakul A (2012) Massive rotator cuff tear associated with acute traumatic posterior shoulder dislocation: report of two cases and literature review. Musculoskelet Surg Jul 11. [Epub ahead of print]

13. Mall NA, Lee AS, Chahal J et al (2013) An evidenced-based examination of the epidemiology and outcomes of traumatic rotator cuff tears. Arthroscopy 29(2):366-376
14. Milgrom C, Schaffler M, Gilbert S, Holsbeeck M van (1995) Rotator-cuff changes in asymptomatic adults. The effect of age, hand dominance and gender. J Bone Joint Surg Br 77(2):296-298

15. Nakajima T, Rokuuma N, Hamada K et al (1994) Histological and biomechanical characteristics of the supraspinatus tendon: reference to rotator cuff tearing. J Shoulder Elbow Surg 3:79-87

16. Namdari S, Henn RF 3rd, Green A (2008) Traumatic anterosuperior rotator cuff tears: the outcome of open surgical repair. J Bone Joint Surg Am 90(9):1906-1913

17. Neer C (1972) Anterior acromioplasty for the chronic impingement syndrome in the shoulder. J Bone Joint Surg Am 54:41-50

18. Nyffeler RW, Werner CM, Sukthankar A et al (2006) Association of a large lateral extension of the acromion with rotator cuff tears. J Bone Joint Surg Am 88(4):800-805

19. Petersen SA, Murphy TP (2011) The timing of rotator cuff repair for the restoration of function. J Shoulder Elbow Surg 20(1):62-68

20. Poppen NK, Walker PS (1978) Forces at the glenohumeral joint in abduction. Clin Orthop Relat Res 135:165-170

21. Prudnikov OE (1994) Simultaneous lesions of the rotator cuff and the brachial plexus. Rev Chir Orthop Reparatrice Appar Mot 80(7):602-609

22. Rickert M, Georgousis H, Witzel U (1998) Native Reißfestigkeit der Sehne des M. supraspinatus beim Menschen - eine biomechanische Untersuchung. Unfallchirurg 101(4):265-270

23. Robinson CM, Shur N, Sharpe T et al (2012) Injuries associated with traumatic anterior glenohumeral dislocations. J Bone Joint Surg Am 94(1):18-26

24. Sano H, Ishii H, Trudel G, Uhthoff HK (1999) Histologic evidence of degeneration at the insertion of 3 rotator cuff tendons: a comparative study with human cadaveric shoulders. J Shoulder Elbow Surg 8(6):574-579

25. Tashjian RZ (2012) Epidemiology, natural history, and indications for treatment of rotator cuff tears. Clin Sports Med 31(4):589-604

26. Weiser L, Assheuer J, Schulitz KP, Castro WH (2012) Magnetic resonance imaging criteria for the differentiation of traumatic and non-traumatic rotator cuff tears. Versicherungsmedizin 64(3):122-126

27. Yadav H, Nho S, Romeo A, MacGillivray JD(2009) Rotator cuff tears: pathology and repair. Knee Surg Sports Traumatol Arthrosc 17(4):409-421

28. Zanetti M, Weishaupt D, Jost B et al (1999) MR imaging for traumatic tears of the rotator cuff: high prevalence of greater tuberosity fractures and subscapularis tendon tears. AJR Am J Roentgenol 172(2):463-467

29. Zuckerman JD, Kummer FJ, Cuomo F et al (1992) The influence of coracoacromial arch anatomy on rotator cuff tears. J Shoulder Elbow Surg 1(1):4-14 